\title{
Pneumonie nach Sportverletzung
}

\section{Korrekte Diagnose erst nach Synkope}

\section{Die plötzlich aufgetretenen Wadenschmerzen eines 44-Jährigen werden zunächst als Muskelfaserruptur interpretiert. Wenige Tage später entwickelt der Mann Husten mit Fieber und röntgenologisch wird eine Pneumonie nachgewiesen. Doch erst als es zu einem synkopalen Ereignis kommt, wird die richtige Diagnose gestellt.}

— Zwei Tage nach dem Besuch des Fitnessstudios stellt sich ein 44-jähriger Mann beim Hausarzt vor und klagt über ziehende Schmerzen im Bereich der linken Wade. Klinisch erscheint die Wade druckschmerzhaft, ansonsten ergeben sich jedoch keine Auffälligkeiten. Bei Verdacht auf einen Muskelfaserriss wird eine topische Therapie mit einer NSAR-haltigen Salbe eingeleitet.

Einige Tage später entwickelt der Patient Fieber bis $40{ }^{\circ} \mathrm{C}$ mit Husten und ziehenden, atemabhängigen, rechtsseitigen Thoraxschmerzen. Die Entzündungsparameter (CRP $24 \mathrm{mg} / \mathrm{dl}$, Leukozyten $14000 / \mu \mathrm{l}$ ) sind deutlich erhöht und röntgenologisch findet sich eine Infiltration im Bereich des rechten Lungenunterfeldes. Es wird eine antibiotische Therapie mit einem Amoxicillinpräparat eingeleitet.

\section{Typischer EKG-Befund}

Drei Tage später klagt der Patient über eine starke plötzlich einsetzende thorakale Beklemmung mit Luftnot, bevor er synkopal wird. Das sofort abgeleitete EKG zeigt einen regelmäßigen Sinusrhythmus mit S1/QIII-Typ mit inkomplettem Rechtsschenkelblock und rechtspräkordial lokalisierten Repolarisationsstörungen vom Innenschichttyp. Dieser Befund spricht eindeutig für eine akute Rechtsherzbelastung im Sinne einer Lungenembolie. Auch echokardiografisch sind die Rechtsherzhöhlen deutlich vergrößert und auch computertomografisch kann die Lungenembolie nachgewiesen werden.

Bei hämodynamischer Stabilität wird keine Thrombolyse durchgeführt, jedoch eine sofortige Antikoagulation mit einem niedermolekularen Heparin in therapeutischer Dosierung überlappend mit Marcumar eingeleitet. Die Kompressionssonografie der Beine zeigt eine nicht mehr ganz frische Thrombose im Bereich der Vena poplitea links.

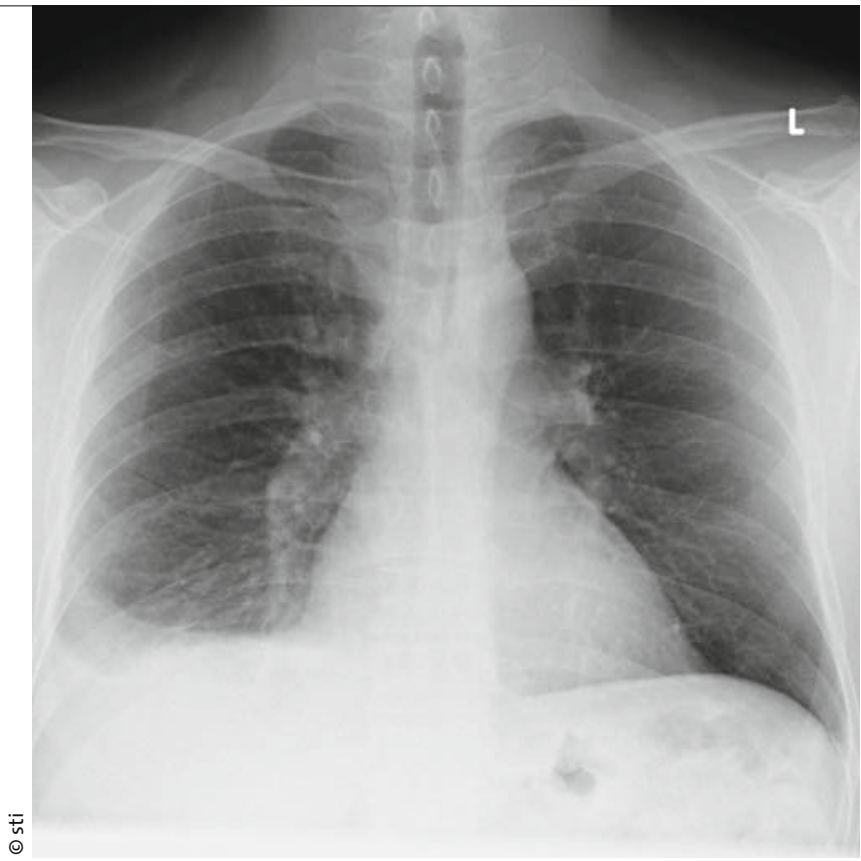

Infiltrationen im rechten Zwerchfellrippenwinkel bei Infarktpneumonie.

\section{Zwei Fehldiagnosen}

Dieser Fall zeigt exemplarisch zwei Fehldiagnosen, die sicherlich keine Rarität sind. So wird eine tiefe Beinvenenthrombose, wenn sie nicht mit einer ausgeprägten typischen Symptomatik einhergeht, häufig zunächst übersehen oder als Muskelfaserriss fehlinterpretiert. Um eine Thrombose bei unspezifischer Symptomatik nicht zu übersehen, empfiehlt sich zumindest die Bestimmung der D-Dimere und evtl. auch die Durchführung einer Kompressionssonografie.

Die zweite Fallgrube ist die Interpretation einer Pneumonie. In den meisten Fällen handelt es sich um eine primäre Pneumonie. Trotzdem sollte immer auch eine sekundäre Ursache diskutiert werden wie z.B. eine Aspirations- oder Infarktpneumonie. Insbesondere bei kleinen peripher gelegenen keilförmigen Verschattungen sollte immer an eine Lungenembolie gedacht werden, denn die richtige pathogenetische Zuordnung der Pneumonie kann für die Patienten lebensrettend sein.

Dr. med. Peter Stiefelhagen 